\title{
Tracheal bullet excreted with the stool: a rare course of events in airway gunshot injury
}

\author{
Nasir U. Din Wani ${ }^{a}$, Tasneem Muzaffar ${ }^{a}$, Syed A. Hussain ${ }^{a}$, Yasir Wani ${ }^{b}$, \\ Danish Zahoor ${ }^{\mathrm{c}}$, Aaliya Wani ${ }^{\mathrm{d}}$, Farhana Bashir ${ }^{\mathrm{e}}$
}

\begin{abstract}
Over the past 10 decades, only five cases have been published of firearm injuries of the chest with conservative management, who in the follow-up expectorated the bullet. We report the first case in the history of gunshot injury chest, where a bullet was found near the posterior-lateral wall of the trachea of the patient who eventually expectorated the bullet with subsequent ingestion and excretion with stools. We try to emphasize the role of repeat morning radiograph in these patients.

Egypt J Bronchol 2019 13:786-787

(C) 2020 Egyptian Journal of Bronchology
\end{abstract}

Egyptian Journal of Bronchology 2019 13:786-787

\section{Introduction}

Spontaneous expectoration of a bullet after penetrating trauma is a rare occurrence and as such has not frequently been detailed in the literature. We describe only the sixth patient in the last 100 years who sustained a gunshot wound to his chest and expectorated the bullet. Our case is the first case in the history where after expectorating the bullet, the patient ingested it with subsequent expulsion with stools.

\section{Case report}

We report a case of an 18-year-old man who sustained a gunshot wound of the right shoulder. He presented to the emergency with complaints of breathlessness. The patient was hemodynamically stable. Chest radiograph was showing a bullet in the neck and right hemopneumothorax. Right-sided chest tube was put in and $200 \mathrm{ml}$ of blood was drained and the patient's symptoms subsided. Non contrast computerised tomography (NCCT) chest and neck was done which was showing bullet in the left lateral wall of the trachea (Fig. 1), right hemopneumothorax with right chest tube in place, pneumomediastinum with subcutaneous emphysema of the chest wall and neck and contusion of the apex of the right lung. Doppler ultrasound of the neck vessels was showing normal major vessels of the neck, normal thyroid, and no significant hematoma. In view of entry wound on the right side and computed tomographic (CT) scan showing bullet in the left lateral wall of the trachea, upper gastrointestinal (GI) endoscopy was done which was normal. In light of the patient being hemodynamically stable without any major vascular injury, the patient was planned for exploration in

\author{
Keywords: firearm injury, tracheal bullet, tracheal gunshot \\ aDepartment of CVTS, Superspeciality Hospital, Shireen Bagh, 'Department \\ of Paediatrics, DHS, 'Department of Microbiology, ${ }^{\mathrm{d}}$ Department of health \\ Services, RNTCP, SKIMS, e'Department of Anaesthesia, GMC, Srinagar, \\ Jammu and Kashmir, India \\ Correspondence to Dr. Nasir U Din Wani, MBBS, MD, MCh CVTS, \\ Superspeciality hospital, Shireen Bagh, KaraNagar, Srinagar, 190010, J\&K, \\ India. \\ e-mail: drnasir629@gmail.com
}

Received: 20 February 2019 Accepted: 26 March 2019

Published: 21 January 2020

elective operation list after 3 days. On the day of surgery, a repeat chest radiograph was advised which was not showing bullet in the neck. The patient was taken for CT scan of the chest, abdomen, and the pelvis which showed a bullet in the cecum. Th patient was taken for repeat upper GI endoscopy to rule out trachea-esophageal fistula, but endoscopy was normal. A flexible bronchoscopy was done, which showed granulation tissue in the left posterior-lateral wall of the trachea seven to eight rings above the carina (Fig. 2). The patient was managed conservatively; chest tube was taken out after 2 days and discharged on the eight day of admission. The patient came to the OPD after 1 week with a preserved bullet. Repeat chest radiograph was done in the OPD which was normal.

\section{Discussion}

Until World War II the mortality of penetrating neck wounds remained at about $15 \%$, which was reduced to $4-7 \%$ by the end of Vietnam war [1]. Till date, only five cases of firearm injuries of the airway which were managed conservatively and eventually expectorated the bullet have been published [2], but this is the first case in which the patient after expectorating the bullet, ingested it, and excreted it with the stool. In all these cases, the patients were hemodynamically stable and a visible bullet was found on chest radiograph. Leslie was the first to describe such a patient in 1917.

This is an open access journal, and articles are distributed under the terms of the Creative Commons Attribution-NonCommercial-ShareAlike 4.0 License, which allows others to remix, tweak, and build upon the work non-commercially, as long as appropriate credit is given and the new creations are licensed under the identical terms. 
Figure 1

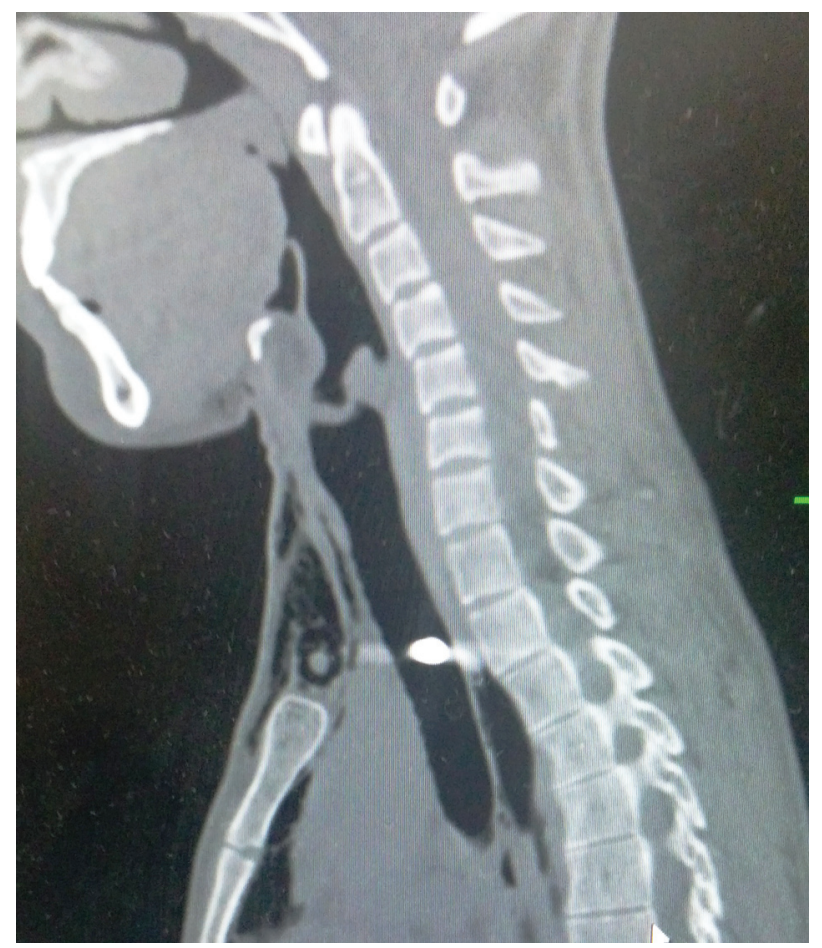

Computed tomographic scan of the neck showing bullet in the lateral wall of the trachea.

The patient was found to have bullet in the right chest, which he spontaneously expectorated after more than 4 months [3]. Andrews and colleagues described a case in which the bullet was found on chest radiograph to be adjacent to the right main bronchus. On the way to the operating room for bronchoscopy, the patient coughed and expectorated the bullet [4]. A similar case was published by Rhodes and Gupta [2] in which the patient had expectorated the bullet while the patient was being taken for bronchoscopy. Hesami and Johari [5] reported a case of a bullet lodged near the tracheal bifurcation. This patient, although stable at presentation, did have a hemopneumothorax and tube thoracostomy was performed. The patient recovered well and was discharged home with the bullet still in place. Three months after the injury, the patient experienced a bout of coughing and expectorated the bullet.

In our patient, on initial evaluation it was difficult to understand the trajectory of the bullet as the entry wound was on the right shoulder and the CT scan was showing a bullet in the left lateral wall of the trachea. Idea of doing upper GI endoscopy and vascular Doppler of the neck was to rule out tracheoesophageal fistula and any injury to major vessels of the neck. With patients' clinical details and other investigations, we can accurately guess the trajectory and course of the bullet in our patient. After entering
Figure 2

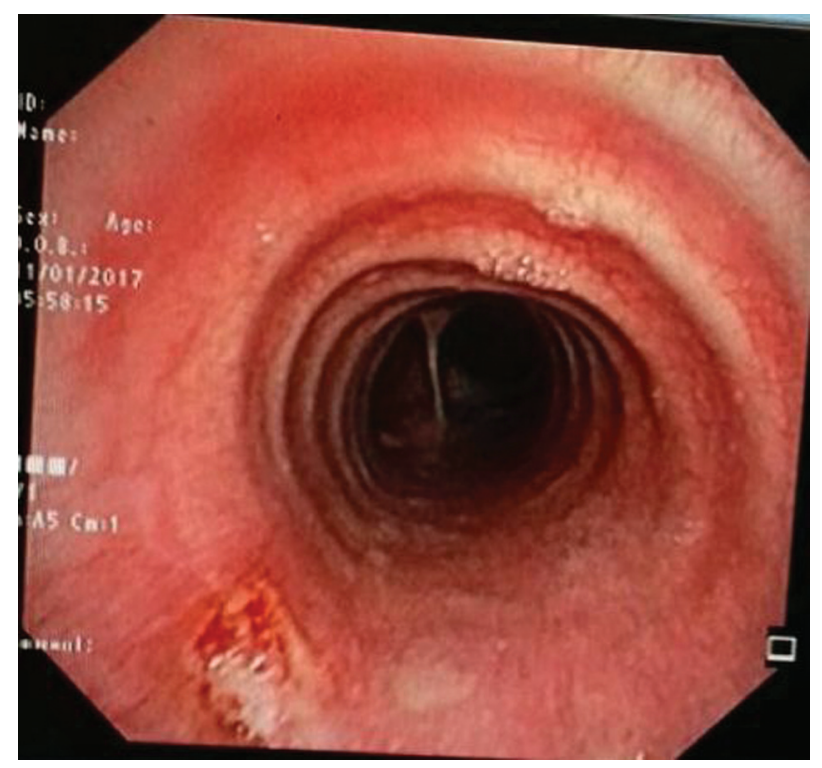

Bronchoscopic image showing granulation tissue in the left posterolateral wall of the trachea.

the right shoulder, the bullet has traversed the right hemithorax causing right lung injury with subsequent right hemopneumothorax and right lung contusion. After that the bullet has pierced the right side of the neck between the trachea and the esophagus and eroded the left posterior-lateral wall of the trachea. Then one day prior to surgery, the bullet has pierced the tracheal lumen and the patient has coughed out the bullet with subsequent ingestion. Here, we try to emphasize the role of bronchoscopy in the evaluation of patients of penetrating neck trauma, and also role of repeat morning radiograph of the affected part to rule out any dislodgement of the foreign body. Morning radiograph of of the neck has saved us from doing undue exploration of the patient.

\section{Financial support and sponsorship}

Nil.

\section{Conflicts of interest}

There are no conflicts of interest.

\section{References}

1 Kochhar LK, Shukul VK, Sharma R. Gunshot wound neck. Indian J Otolaryngol Head Neck Surg 2004; 56:49-50.

2 Rhodes SC, Gupta SS. Expectoration of a bullet after gunshot wound to the chest. J Emerg Trauma Shock 2013; 6:135-137.

3 Leslie RM. Spontaneous evacuation of a shrapnel bullet in the lung by expectoration. Br Med J 1917; 2:648-649.

4 Andrews CM, Singh NN, Stewart RW. Bullet aspiration and spontaneous expectoration after gunshot wound to trachea. Mil Med 2010; 175:72-73.

5 Hesami SM, Johari HG. Spontaneous expectoration of an asymptomatic retained intrathoracic bullet. Injury 2012; 43: 1219-1221. 